\title{
Examples of foliations with infinite dimensional special cohomology
}

\author{
Andrzej Czarnecki ${ }^{1}$ ( Paweł Raźny ${ }^{1}$
}

Received: 5 May 2017 / Accepted: 14 July 2017 / Published online: 2 August 2017

(C) The Author(s) 2017. This article is an open access publication

\begin{abstract}
We present two examples of foliations with infinite dimensional basic symplectic and complex cohomologies, along with a general sufficient condition for such phenomena. This puts restrictions on possible generalisations of several finiteness results from Riemannian foliations to any broader class. The examples are also noteworthy for the unusual behaviour of their basic de Rham cohomology.
\end{abstract}

Keywords Foliations · Transverse structures · Basic cohomology

Mathematics Subject Classification $53 \mathrm{C} 12 \cdot 57 \mathrm{R} 18$

\section{Introduction}

In this short paper, a simple algebraic Lemma 1 (based on the methods used in [4]) enables us to relate the special basic cohomologies to the ordinary basic cohomology of a foliation. We thus obtain interesting examples of infinite dimensional basic Bott-Chern and Aeppli cohomologies (in the transversally holomorphic case), and basic $\left(d+d^{\Lambda}\right)$ - and $d d^{\Lambda}$-cohomologies (in the transversally symplectic case). These cohomologies (and especially their non-foliated counterparts) are subject of extensive studies (cf. $[2-4,9,12,14]$ ); in particular, it can be proved (cf. [12]) that for Riemannian foliations they are all finite dimensional. Our examples amount to say that certain compactness conditions in those proofs cannot be dropped, which is by no means obvious (cf. [10], which works for both compact and non-compact manifolds). The examples also violate various dualities present in the Riemannian case. We will begin the next section with a review of all relevant notions.

\footnotetext{
$凶$ Andrzej Czarnecki

andrzejczarnecki01@gmail.com

Paweł Raźny

pawel.razny@student.uj.edu.pl

1 Jagiellonian University, Łojasiewicza 6, 30-348 Kraków, Poland
} 
The behaviour of special basic cohomologies in our examples revealed an interesting picture. Of all the peculiarities collected in the last section, we single out here the following: The basic cohomology of our transversally holomorphic example has infinite dimension in degrees 2 and 4, and finite dimension in degree 3. To the extent of our knowledge, no example of basic cohomology reverting to finite dimension was ever given. It is worth pointing out that we know essentially only two ways of producing infinite dimensional basic cohomology: either Schwarz' [13] or Ghys' [7]. We feel it is important to better understand how the infinite dimensional basic cohomology may arise in non-Riemannian foliations. Lemma 1 and its application to the transversally holomorphic example shed some light on this matter.

\section{Foliations}

\subsection{Transverse structures}

We start with a brief review of some basic facts about foliations and transverse structures. The interested reader is referred to [11] for a thorough exposition. All manifolds are assumed to be compact.

Definition 1 A codimension $n$ foliation $\mathcal{F}$ on a smooth manifold $M$ is given by the following data:

- an open cover $\mathcal{U}:=\left\{U_{i}\right\}_{i \in I}$ of $\mathbf{M}$;

- a $n$-dimensional smooth manifold $T_{0}$;

- for each $U_{i} \in \mathcal{U}$ a submersion $f_{i}: U_{i} \longrightarrow T_{0}$ with connected fibres (called plaques);

- for each intersection $U_{i} \cap U_{j} \neq \emptyset$ a local diffeomorphism $\gamma_{i j}$ of $T_{0}$ such that $f_{j}=\gamma_{i j} \circ f_{i}$.

The last condition ensures that the plaques glue nicely to form a partition of $M$ by submanifolds of codimension $n$, called leaves of $\mathcal{F}$. We call $T=\bigsqcup_{U_{i} \in \mathcal{U}} f_{i}\left(U_{i}\right)$ the transverse manifold of $\mathcal{F}$. The local diffeomorphisms $\gamma_{i j}$ generate a pseudogroup $\Gamma$ of transformations on $T$ (called the holonomy pseudogroup).

The space of leaves $M / \mathcal{F}$ of the foliation $\mathcal{F}$ can be identified with $T / \Gamma$. We note that neither $T$ nor $T / \Gamma$ need to be compact, even if $M$ is.

Definition 2 A smooth form $\omega$ on $M$ is called basic iff for any vector field $X$ tangent to the leaves of $\mathcal{F}$ we have

$$
i_{X} \omega=i_{X} \mathrm{~d} \omega=0
$$

Basic forms are in one-to-one correspondence with $\Gamma$-invariant smooth forms on $T$, a point of view that we will take below.

It is clear that $d \omega$ is basic for any basic form $\omega$. Hence, the set of basic forms of $\mathcal{F}$, $\Omega^{\bullet}(M / \mathcal{F})$, is a subcomplex of the de Rham complex of $M$. We define the basic cohomology (or sometimes basic de Rham cohomology if other basic cohomologies are in play) of $\mathcal{F}$ to be the cohomology of this subcomplex and denote it by $H^{\bullet}(M / \mathcal{F})$.

A transverse structure on $\mathcal{F}$ is any $\Gamma$-invariant structure on $T$. We will need the following examples.

Definition $3 \mathcal{F}$ is said to be transversally orientable iff $T$ is orientable and all the $\gamma_{i j}$ are orientation preserving. $\mathcal{F}$ is said to be homologically orientable if the top basic cohomology space $H^{n}(M / \mathcal{F})=\mathbb{R}$. Contrary to the non-foliated case, these two notions are not equivalent as we will see later on. 
Definition $4 \mathcal{F}$ is said to be Riemannian iff $T$ has a $\Gamma$-invariant Riemannian metric.

We emphasise here a strong property of the Riemannian foliations: their basic cohomology is always finite dimensional. We refer the reader to [6] for the proof applicable also to the other cohomologies considered below.

From now on, we will restrict our attention to foliations of even codimension $2 n$.

Definition $5 \mathcal{F}$ is said to be transversally symplectic iff $T$ admits a $\Gamma$-invariant closed non-degenerate 2-form $\omega$, called a transverse symplectic form. Again, contrary to the nonfoliated case, this does not imply homological orientability, although it makes $\mathcal{F}$ transversally oriented.

Definition $6 \mathcal{F}$ is said to be transversally holomorphic iff $T$ admits a complex structure that makes all the $\gamma_{i j}$ holomorphic.

Definition 7 A foliation is said to be Hermitian if it is both transversally holomorphic and Riemannian (we emphasise that neither of these condition implies the other), and both structures are compatible. The compatibility condition is not restrictive: given any Riemannian structure $g$ and transverse holomorphic structure $J, \frac{g(\cdot, \cdot)+g(J \cdot, J \cdot)}{2}$ is a Riemannian structure compatible with $J$.

If $\mathcal{F}$ is transversally holomorphic, we have the standard decomposition of the space of complex-valued forms $\Omega^{\bullet}(M / \mathcal{F}, \mathbb{C})$ into forms of the type $(p, q)$, and $d$ decomposes as $\partial+\bar{\partial}$ of orders $(1,0)$ and $(0,1)$, respectively. We can then define the basic Dolbeault double complex $\left(\Omega^{\bullet \bullet \bullet}(M / \mathcal{F}, \mathbb{C}), \partial, \bar{\partial}\right)$, basic Dolbeault cohomology $H_{\bar{\partial}}^{\bullet}(M / \mathcal{F})=\operatorname{ker} \bar{\partial} / \operatorname{im} \bar{\partial}$, and basic Frölicher spectral sequence just like in the non-foliated case. We note that for a Hermitian foliation, the basic Dolbeault cohomology is again finite dimensional and even without this assumption, the basic Frölicher sequence converges to the basic cohomology.

\subsection{Bott-Chern and Aeppli cohomology theories}

Let $M$ be a manifold endowed with a transversally holomorphic foliation $\mathcal{F}$ of complex codimension $n$. Using the operators $\partial$ and $\bar{\partial}$ above, we can construct the basic Bott-Chern and basic Aeppli cohomologies of $\mathcal{F}$ :

$$
\begin{aligned}
H_{B C}^{\bullet \bullet \bullet}(M / \mathcal{F}) & :=\frac{\operatorname{ker} \partial \cap \operatorname{ker} \bar{\partial} \cap \Omega^{\bullet \bullet \bullet}(M / \mathcal{F})}{\operatorname{im} \partial \bar{\partial} \cap \Omega^{\bullet, \bullet}(M / \mathcal{F})} \\
H_{A}^{\bullet \bullet \bullet}(M / \mathcal{F}) & :=\frac{\operatorname{ker} \partial \bar{\partial} \cap \Omega^{\bullet \bullet \bullet}(M / \mathcal{F})}{\left.\operatorname{im} \partial \cap \operatorname{im} \bar{\partial} \cap \Omega^{\bullet, \bullet}(M / \mathcal{F})\right)}
\end{aligned}
$$

For a non-foliated account of these cohomologies, see [1]. Assuming the foliation is Hermitian, we have some restrictions on these cohomologies. We recall the results from [12].

Theorem 1 If $M$ is a compact manifold endowed with a Hermitian foliation $\mathcal{F}$, then the dimensions of $H_{B C}^{\bullet, \bullet}(M / \mathcal{F})$ and $H_{A}^{\bullet, \bullet}(M / \mathcal{F})$ are finite.

Corollary 1 If M is a compact manifold endowed with a Hermitian homologically orientable foliation $\mathcal{F}$, then there is an isomorphism

$$
H_{B C}^{p, q}(M / \mathcal{F}) \longrightarrow H_{A}^{n-p, n-q}(M / \mathcal{F})
$$

induced by the transverse Hodge star operator. 
Note that these theorems apply also to any transversally holomorphic Riemannian foliation since we can tweak the given Riemannian structure to a Hermitian one, as described in Definition 7. Neither Bott-Chern, nor Aeppli cohomology depends on the metric, and the theorem only requires any Hermitian metric to be present.

We also note that for a non-foliated Kähler manifold, these cohomologies are isomorphic to Dolbeault cohomology - so their failure to do so measures how far a manifold is from being Kähler. The same applies to the foliated, transversally Kähler case.

\section{$2.3 d d^{\Lambda}$ - and $\left(d+d^{\Lambda}\right)$-cohomology theories}

Let $\mathcal{F}$ be a transversally symplectic foliation of codimension $2 n$ on $M$ with a basic symplectic form $\omega$. Let us start with the definition of the symplectic star operator for $\mathcal{F}$. The transverse symplectic form defines a non-degenerate pairing $\tilde{G}$ of the vector fields on the transverse manifold. We can then extend it to a (non-degenerate) pairing $G$ on basic forms.

Definition 8 The symplectic star operator is a linear operator

$$
*_{s}: \Omega^{k}(M / \mathcal{F}) \longrightarrow \Omega^{2 n-k}(M / \mathcal{F})
$$

uniquely defined by the formula

$$
\alpha_{1} \wedge *_{s} \alpha_{2}=G\left(\alpha_{1}, \alpha_{2}\right) \frac{\omega^{n}}{n !}
$$

where $\alpha_{1}$ and $\alpha_{2}$ are arbitrary basic $k$-forms.

The symplectic star operator indeed preserves the basic forms and is an isomorphism, cf. $[5,10]$ for the theory in the non-foliated case. Out of the many operators connected to the symplectic star, we only use

$$
d^{\Lambda} \alpha:=(-1)^{k+1} *_{s} d *_{s}(\alpha)
$$

where $\alpha$ is again any basic $k$-form. We note the relation $d d^{\Lambda}+d^{\Lambda} d=0$. We can use this operator to define basic cohomology theories similar to those reviewed in the previous subsection (cf. [14]):

$$
\begin{aligned}
H_{d^{\Lambda}}^{\bullet}(M / \mathcal{F}) & :=\frac{\operatorname{ker}\left(d^{\Lambda}\right)}{\operatorname{im}\left(d^{\Lambda}\right)} \\
H_{d^{\circ} d^{\Lambda}}^{\bullet}(M / \mathcal{F}) & :=\frac{\operatorname{ker}\left(d+d^{\Lambda}\right)}{\operatorname{im}\left(d d^{\Lambda}\right)} \\
H_{d^{\Lambda}}^{\bullet}(M / \mathcal{F}) & :=\frac{\operatorname{ker}\left(d d^{\Lambda}\right)}{\operatorname{im}(d)+\operatorname{im}\left(d^{\Lambda}\right)}
\end{aligned}
$$

It is easy to see that the basic $d^{\Lambda}$-cohomology is simply the basic cohomology with reversed gradation and hence will not concern us. In a manner slightly different from Corollary 1 , the symplectic star gives Poincaré dualities in the last two cohomologies

$$
\begin{gathered}
H_{d d^{\Lambda}}^{p}(M / \mathcal{F}) \longrightarrow H_{d d^{\Lambda}}^{2 n-p}(M / \mathcal{F}) \\
H_{d+d^{\Lambda}}^{p}(M / \mathcal{F}) \longrightarrow H_{d+d^{\Lambda}}^{2 n-p}(M / \mathcal{F})
\end{gathered}
$$

We note that this does not depend on the presence of neither the transverse Riemannian structure (which may well not exist) nor the homological orientation (cf. Definitions 3 and 5). 
It can be shown using $[12,14]$ that for a Riemannian transversally symplectic foliation $H_{d^{\Lambda}}^{\bullet}$ and $H_{d+d^{\Lambda}}^{\bullet}$ are finite dimensional.

\section{An algebraic lemma and its consequences}

Let $I_{1}, I_{2}, I_{12}, K_{1}, K_{2}, K_{12}$ be vector spaces satisfying

$$
I_{12} \subset I_{1}, I_{2} \quad I_{1} \subset K_{1} \quad I_{2} \subset K_{2} \quad K_{1}, K_{2} \subset K_{12}
$$

Then, the following lemma holds:

Lemma 1 If $K_{1} / I_{1}$ or $K_{2} / I_{2}$ have infinite dimension, then $\left(K_{1} \cap K_{2}\right) / I_{12}$ or $K_{12} /\left(I_{1}+I_{2}\right)$ has infinite dimension as well.

Proof Without loss of generality, let us assume that $K_{1} / I_{1}$ is infinite dimensional. Then, there are two sequences

$$
\begin{aligned}
& \left(K_{1} \cap I_{2}\right) / I_{12} \stackrel{f^{\prime}}{\longrightarrow} K_{1} / I_{1} \stackrel{g^{\prime}}{\longrightarrow} K_{12} /\left(I_{1}+I_{2}\right) \\
& \left(K_{1} \cap K_{2}\right) / I_{12} \stackrel{f^{\prime \prime}}{\longrightarrow} K_{1} / I_{1} \stackrel{g^{\prime \prime}}{\longrightarrow} K_{12} /\left(I_{1}+K_{2}\right)
\end{aligned}
$$

It is easy to see that these sequences are exact in the middle term (since, for example, the appropriate kernel and image are classes represented by elements of $\left.\left(K_{1} \cap I_{2}\right)\right)$. If both $\left(K_{1} \cap K_{2}\right) / I_{12}$ and $K_{12} /\left(I_{1}+I_{2}\right)$ have finite dimension, then so do $\left(K_{1} \cap I_{2}\right) / I_{12}$ and $K_{12} /\left(I_{1}+K_{2}\right)$ since they are smaller. But then the middle term has finite dimension by exactness, a contradiction.

We can apply this lemma to the transversally symplectic and transversally holomorphic structures to get

Proposition 1 If $\mathcal{F}$ is a transversally symplectic foliation for which $H^{k}(M / \mathcal{F})$ is infinite dimensional, then $H_{d+d^{\Lambda}}^{k}(M / \mathcal{F})$ or $H_{d d^{\Lambda}}^{k}(M / \mathcal{F})$ is infinite dimensional as well.

Proof In the lemma take:

$$
\begin{array}{lll}
K_{1}=\operatorname{ker} d & K_{2}=\operatorname{ker} d^{\Lambda} & K_{12}=\operatorname{ker} d d^{\Lambda} \\
I_{1}=\operatorname{im} d & I_{2}=\operatorname{im} d^{\Lambda} & I_{12}=\operatorname{im} d d^{\Lambda}
\end{array}
$$

Proposition 2 If $\mathcal{F}$ is a transversally holomorphic foliation for which $H_{\bar{\partial}}^{p, q}(M / \mathcal{F})$ is infinite dimensional, then $H_{B C}^{p, q}(M / \mathcal{F})$ or $H_{A}^{p, q}(M / \mathcal{F})$ is infinite dimensional as well.

Proof In the lemma take:

$$
\begin{aligned}
K_{1}=\operatorname{ker} \partial & K_{2}=\operatorname{ker} \bar{\partial} & K_{12}=\operatorname{ker} \partial \bar{\partial} \\
I_{1}=\operatorname{im} \partial & I_{2}=\operatorname{im} \bar{\partial} & I_{12}=\operatorname{im} \partial \bar{\partial}
\end{aligned}
$$


Corollary 2 If $\mathcal{F}$ is a transversally holomorphic foliation such that $H^{k}(M / \mathcal{F})$ has infinite dimension, then for some $(p, q)$ satisfying $p+q=k, H_{B C}^{p, q}(M / \mathcal{F})$ or $H_{A}^{p, q}(M / \mathcal{F})$ has infinite dimension.

Proof By the previous proposition, it is sufficient to prove that Dolbeault cohomology has infinite dimension for some $(p, q)$ with $p+q=k$. This is obvious since the basic Frölicher spectral sequence converges to the basic cohomology of $\mathcal{F}$ and so the dimensions of the entries on the first page must be greater than those in the limit.

\section{Transversally symplectic example}

Consider, as in [8], a map of the 2-torus $\mathbb{T}^{2}$ given by the matrix $A=\left[\begin{array}{ll}1 & 1 \\ 0 & 1\end{array}\right]$. We form a suspension of this map, $\left(M, \mathcal{F}_{A}\right)$ : a codimension two foliation on $\mathbb{T}^{2} \times[0,1] /(t, 0) \sim(A t, 1)$. The plaques of this foliation are the lines $\left\{t_{0}\right\} \times[0,1]$ and $\mathbb{T}^{2}$ can be taken for the transverse manifold, with the infinite cyclic group generated by $A$ as the holonomy pseudogroup. Since $\operatorname{det} A=1$, this foliation is transversally symplectic with the standard symplectic form $d x \wedge d y$ on $\mathbb{T}^{2}$.

We determine the basic complex. Any basic function $f$ must satisfy $f(x, y)=f(x+y, y)$. Taking an irrational $y_{0}$ and any $x_{0}$, we see that $f$ does not depend on the first coordinate, since it is constant on $\left\{\left(x_{0}+n y_{0}, y_{0}\right)\right\}$, dense in $\left\{\left(x, y_{0}\right)\right\}$. Therefore, the basic functions correspond to smooth functions on a circle

$$
\Omega^{0}\left(M / \mathcal{F}_{A}\right)=\left\{f(y) \mid f \in \mathcal{C}^{\infty}\left(\mathbb{S}^{1}\right)\right\}
$$

In a similar fashion, we see that

$$
\begin{aligned}
& \Omega^{1}\left(M / \mathcal{F}_{A}\right)=\left\{f(y) \mathrm{d} y \mid f \in \mathcal{C}^{\infty}\left(\mathbb{S}^{1}\right)\right\} \\
& \Omega^{2}\left(M / \mathcal{F}_{A}\right)=\left\{f(y) \mathrm{d} x \wedge \mathrm{d} y \mid f \in \mathcal{C}^{\infty}\left(\mathbb{S}^{1}\right)\right\}
\end{aligned}
$$

It is then easy to see that the basic cohomology is

1. $H^{0}\left(M / \mathcal{F}_{A}\right)=H^{1}\left(M / \mathcal{F}_{A}\right)=\mathbb{R}$

2. $H^{2}\left(M / \mathcal{F}_{A}\right)=\mathcal{C}^{\infty}\left(\mathbb{S}^{1}\right)$

We note again that this precludes $\mathcal{F}_{A}$ from being Riemannian.

We compute $H_{d_{+d^{\Lambda}}}^{\bullet}\left(M / \mathcal{F}_{A}\right)$ and $H_{d^{\Lambda}}^{\bullet}\left(M / \mathcal{F}_{A}\right)$. Observe that $d d^{\Lambda}=-d^{\Lambda} d=0$, because in degree 2 and 0 it factors through the trivial spaces, $\Omega^{3}\left(M / \mathcal{F}_{A}\right)$ and $\Omega^{-1}(M /$ $\mathcal{F}_{A}$ ), respectively, and in degree $1, d d^{\Lambda} f(y) d y=-d^{\Lambda} d f(y) d y=0$ (or because $*_{s}=i d$ on $\Omega^{1}\left(M / \mathcal{F}_{A}\right)$ ). Consequently

1. $H_{d+d^{\Lambda}}^{0}\left(M / \mathcal{F}_{A}\right)=H_{d+d^{\Lambda}}^{2}\left(M / \mathcal{F}_{A}\right)=\mathbb{R}$

2. $H_{d+d^{\Lambda}}^{1}\left(M / \mathcal{F}_{A}\right)=\mathcal{C}^{\infty}\left(\mathbb{S}^{1}\right)$

and

1. $H_{d d^{\Lambda}}^{0}\left(M / \mathcal{F}_{A}\right)=H_{d d^{\Lambda}}^{2}\left(M / \mathcal{F}_{A}\right)=\mathcal{C}^{\infty}\left(\mathbb{S}^{1}\right)$

2. $H_{d d^{\Lambda}}^{1}\left(M / \mathcal{F}_{A}\right)=\mathbb{R}$ 


\section{Transversally holomorphic example}

To provide a transversally holomorphic foliation exhibiting a similar behaviour, we mimic the construction presented above. We take the map of the 4-torus $\mathbb{T}^{4}$ induced by the matrix $A=\left[\begin{array}{llll}1 & 0 & 1 & 0 \\ 0 & 1 & 0 & 1 \\ 0 & 0 & 1 & 0 \\ 0 & 0 & 0 & 1\end{array}\right]$. As before, we form a suspension of this map, $\left(M, \mathcal{F}_{A}\right):$ a codimension four foliation on $\mathbb{T}^{4} \times[0,1] /(t, 0) \sim(A t, 1)$. Since $A$ is in $G l(2, \mathbb{C}) \subset G l(4, \mathbb{R})$, this foliation is transversally holomorphic with the complex structure induced from $\mathbb{T}^{4}$.

On the transverse manifold $\mathbb{T}^{4}$, we will use real coordinates $\left(x_{1}, y_{1}, x_{2}, y_{2}\right)$ (better suited for the suspension) and then switch to complex coordinates $(w, z)=\left(x_{1}+i y_{1}, x_{2}+i y_{2}\right)$ (better suited for the bigradation of the complex forms). We will describe the $A$-invariant forms, computing only the 2 -forms explicitly as an example. The operators $\partial, \bar{\partial}$ and $\partial \bar{\partial}$ will prove to be not too complicated, and we will proceed to compute basic de Rham, Dolbeault, Aeppli and Bott-Chern cohomologies.

\subsection{Invariant forms}

As in the previous example, we can easily see the invariant complex functions to depend only on the last two real coordinates, or on the complex coordinate $z$. Hence, $\Omega^{0}\left(M / \mathcal{F}_{A}, \mathbb{C}\right)=$ $\mathcal{C}^{\infty}\left(\mathbb{T}^{2}, \mathbb{C}\right)$.

An $A$-invariant complex 2-form on the 4-torus is a section of $\bigwedge^{2} T^{*}\left(\mathbb{T}^{4}\right)$; therefore, a skew-symmetric matrix

$$
\begin{aligned}
& \alpha_{\left(x_{1}, y_{1}, x_{2}, y_{2}\right)} \\
& \quad=\left[\begin{array}{cccc}
0 & f_{1}\left(x_{1}, y_{1}, x_{2}, y_{2}\right) & f_{2}\left(x_{1}, y_{1}, x_{2}, y_{2}\right) & f_{3}\left(x_{1}, y_{1}, x_{2}, y_{2}\right) \\
-f_{1}\left(x_{1}, y_{1}, x_{2}, y_{2}\right) & 0 & f_{4}\left(x_{1}, y_{1}, x_{2}, y_{2}\right) & f_{5}\left(x_{1}, y_{1}, x_{2}, y_{2}\right) \\
-f_{2}\left(x_{1}, y_{1}, x_{2}, y_{2}\right) & -f_{4}\left(x_{1}, y_{1}, x_{2}, y_{2}\right) & 0 & f_{6}\left(x_{1}, y_{1}, x_{2}, y_{2}\right) \\
-f_{3}\left(x_{1}, y_{1}, x_{2}, y_{2}\right) & -f_{5}\left(x_{1}, y_{1}, x_{2}, y_{2}\right) & -f_{6}\left(x_{1}, y_{1}, x_{2}, y_{2}\right) & 0
\end{array}\right]
\end{aligned}
$$

Since it is invariant, it satisfies

$$
\alpha_{\left(x_{1}, y_{1}, x_{2}, y_{2}\right)}=A^{t} \alpha_{A\left(x_{1}, y_{1}, x_{2}, y_{2}\right)} A
$$

that amounts to $f_{1}=0$ and $f_{3}=f_{4}$, and all the functions being $A$-invariant. This gives

$$
\begin{aligned}
\Omega^{2}\left(M / \mathcal{F}_{A}, \mathbb{C}\right)= & \left\{f_{2}\left(x_{2}, y_{2}\right) d x_{1} \wedge d x_{2}\right\} \\
& \oplus\left\{f_{3}\left(x_{2}, y_{2}\right)\left(d x_{1} \wedge d y_{2}+d y_{1} \wedge d x_{2}\right)\right\} \\
& \oplus\left\{f_{5}\left(x_{2}, y_{2}\right) d y_{1} \wedge d y_{2}\right\} \\
& \oplus\left\{f_{6}\left(x_{2}, y_{2}\right) d x_{2} \wedge d y_{2}\right\}
\end{aligned}
$$

which we will now rewrite in the complex coordinates

$$
\begin{aligned}
\Omega^{2}\left(M / \mathcal{F}_{A}, \mathbb{C}\right)= & \{b(z) d w \wedge d z\} \\
& \oplus\{c(z)(d w \wedge d \bar{z}+d \bar{w} \wedge d z)\} \\
& \oplus\{e(z) d \bar{w} \wedge d \bar{z}\} \\
& \oplus\{f(z) d z \wedge d \bar{z}\}
\end{aligned}
$$

Note that any complex function of the complex coordinate is to be smooth, not holomorphic. 


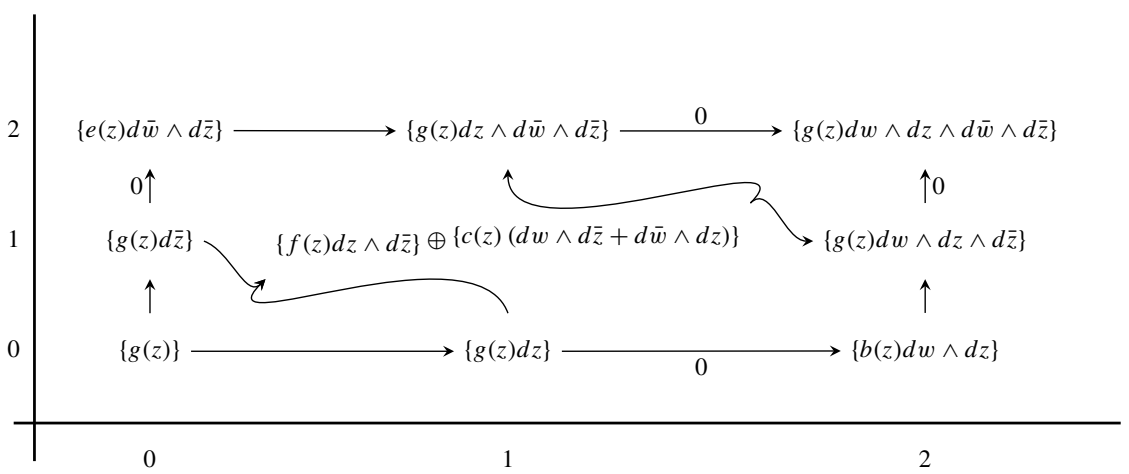

We present all the invariant forms with the complex bigradation $\Omega^{\bullet \bullet \bullet}\left(M / \mathcal{F}_{A}, \mathbb{C}\right)$, indicating where the differentials are obviously trivial. We use a generic letter $g$ for functions in degrees other than 2, since the labelling will play no role there. The curvy arrows are meant to indicate that $d \Omega^{1}\left(M / \mathcal{F}_{A}\right)$ is contained in the $\{f(z) d z \wedge d \bar{z}\}$ term of $\Omega^{1,1}\left(M / \mathcal{F}_{A}\right)$ and $d \Omega^{1,1}(M /$ $\left.\mathcal{F}_{A}\right)=d\{c(z)(d w \wedge d \bar{z}+d \bar{w} \wedge d z)\}$. Note that the diagram shows that $\partial \bar{\partial}$ can be nonzero only on the 0 -forms.

\subsection{De Rham cohomology}

We compute the basic cohomology over $\mathbb{C}$. Some of the spaces involved can be described in terms of cohomology of the complex torus $\mathbb{T}^{2}$-parts of the diagram above clearly repeat parts of $\Omega^{\bullet \bullet \bullet}\left(\mathbb{T}^{2}, \mathbb{C}\right)$-which is not complicated since the torus is Kähler.

Since the differentials are quite simple too, we hope that the reader will have no trouble justifying the claims below.

1. $H^{0}\left(M / \mathcal{F}_{A}\right) \simeq H^{0}\left(\mathbb{T}^{2}\right)=\mathbb{C}$;

2. $H^{1}\left(M / \mathcal{F}_{A}\right) \simeq H^{1}\left(\mathbb{T}^{2}\right)=\mathbb{C}^{2}$;

3. $H^{2}\left(M / \mathcal{F}_{A}\right) \simeq V \oplus H^{2}\left(\mathbb{T}^{2}\right)=V \oplus \mathbb{C}$, where $V$ is an infinite dimensional space $\{\bar{\partial} b-$ $\partial c=\bar{\partial} c-\partial e=0\}$ easily seen to be infinite dimensional; none of these closed forms is exact since the image $d \Omega^{1}\left(M / \mathcal{F}_{A}\right) \subset\{f(z) d z \wedge d \bar{z}\}$; the term $H^{2}\left(\mathbb{T}^{2}\right)$ follows from this inclusion;

4. $H^{3}\left(M / \mathcal{F}_{A}\right) \simeq\left(H^{2}\left(\mathbb{T}^{2}\right)\right)^{2}=\mathbb{C}^{2}$ since the $d w$ and $d \bar{w}$ factors do not interfere in any way;

5. $H^{4}=\mathcal{C}^{\infty}\left(\mathbb{T}^{2}, \mathbb{C}\right)$ since the image $d \Omega^{3}\left(M / \mathcal{F}_{A}\right)$ is trivial;

We present the three complex cohomologies in diagrams explaining their entries below each one. 


\subsection{Dolbeault cohomology}

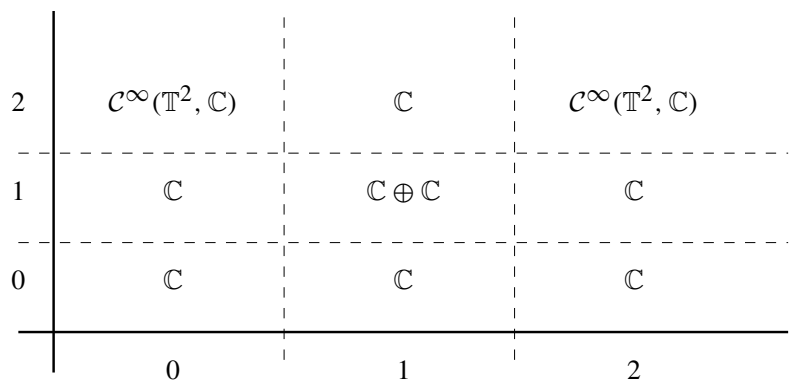

1. $H_{\bar{\partial}}^{0,0}$ is represented by the constant functions;

2. $H_{\bar{\partial}}^{1,0}$ and $H_{\bar{\partial}}^{2,0}$ are represented by the holomorphic functions;

3. $H_{\bar{\partial}}^{1,1}$ splits as $H_{\bar{\partial}}^{1,1}\left(\mathbb{T}^{2}\right)$ and the holomorphic functions;

4. $H_{\bar{\partial}}^{0,1}$ is again identified with the holomorphic functions-note that $\bar{\partial}\{g(z)\}$ is isomorphic to the space of all functions divided by the holomorphic ones, and we take a quotient again to get $H_{\bar{\partial}}^{0,1}=\mathcal{C}^{\infty}\left(\mathbb{T}^{2}, \mathbb{C}\right) /\left(\mathcal{C}^{\infty}\left(\mathbb{T}^{2}, \mathbb{C}\right) / \mathbb{C}\right)=\mathbb{C}$; the same reasoning applies to $H_{\bar{\partial}}^{2,1}$ and $H_{\bar{\partial}}^{1,2}$;

5. the remaining spaces are $\mathcal{C} \infty\left(\mathbb{T}^{2}, \mathbb{C}\right)$ since the relevant differentials are all trivial.

\subsection{Bott-Chern cohomology}

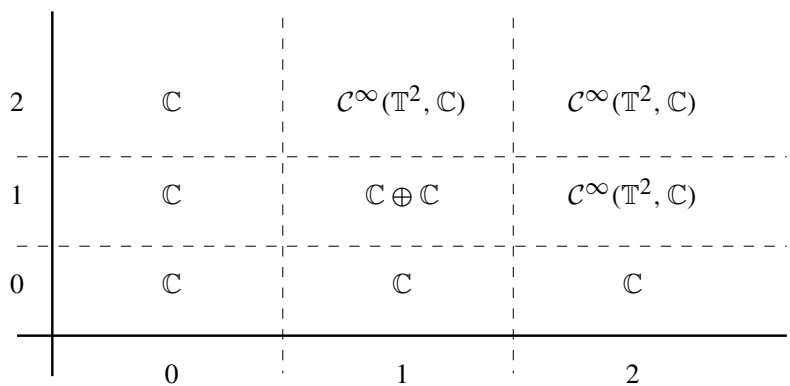

1. $H_{B C}^{0,0}$ is represented by the constant functions;

2. $H_{B C}^{1,0}$ and $H_{B C}^{0,1}$ are represented by the holomorphic and antiholomorphic functions, respectively;

3. so are $H_{B C}^{2,0}$ and $H_{B C}^{0,2}$;

4. $H_{B C}^{1,1}$ is $H_{B C}^{1,1}\left(\mathbb{T}^{2}\right)$ plus the constant functions coming from the second summand in $\Omega^{1,1}\left(M / \mathcal{F}_{A}\right)$

5. $H_{B C}^{2,1}=H_{B C}^{1,2}=H_{B C}^{2,2}=\mathcal{C}^{\infty}\left(\mathbb{T}^{2}, \mathbb{C}\right)$ since each of the $\partial, \bar{\partial}$, and $\partial \bar{\partial}$ is trivial in these cases. 


\subsection{Aeppli cohomology}

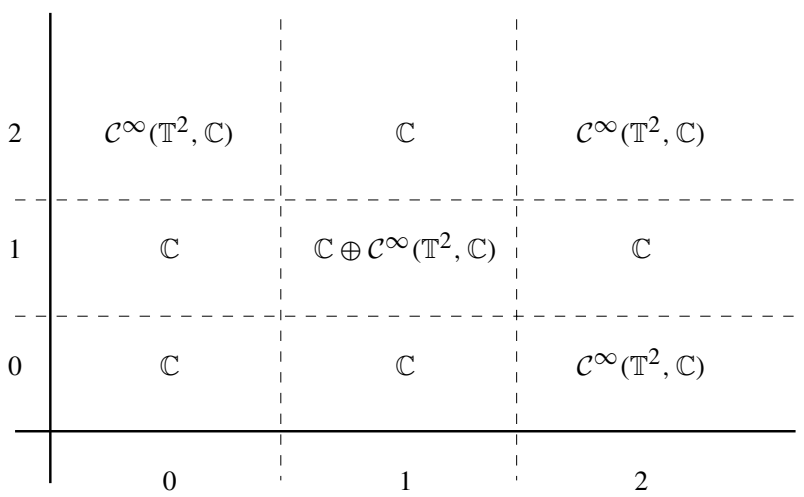

1. $H_{A}^{0,0}$ is represented by constant functions;

2. $H_{A}^{1,0}=H_{A}^{1,0}\left(\mathbb{T}^{2}\right)$ and $H_{A}^{0,1}=H_{A}^{0,1}\left(\mathbb{T}^{2}\right)$;

3. $H_{A}^{2,0}$ and $H_{A}^{0,2}$ are all the relevant forms, since the differentials are all zero in these cases;

4. $H_{A}^{1,1}$ splits into $H_{B C}^{1,1}\left(\mathbb{T}^{2}\right)=H^{2}\left(\mathbb{T}^{2}\right)$ plus $\mathcal{C}^{\infty}\left(\mathbb{T}^{2}, \mathbb{C}\right)$ since the $\partial \bar{\partial}$ is trivial;

5. $H_{A}^{2,1}=H_{A}^{1,2}$ is again $H_{A}^{1,2}\left(\mathbb{T}^{2}\right)=H_{A}^{2,1}\left(\mathbb{T}^{2}\right)$;

6. $H_{A}^{2,2}=\mathcal{C}^{\infty}\left(\mathbb{T}^{2}, \mathbb{C}\right)$ since all the differentials are trivial.

\section{Conclusions}

We close this paper summarising some interesting properties of the given examples.

Remark 1 The transversally symplectic example highlights that the infinite dimension of symplectic cohomology in dimension $k$ may stem, by Proposition 1 , from the infinite dimension of the de Rham basic cohomology in degree $k\left(H_{d d^{\Lambda}}^{2}\left(M / \mathcal{F}_{A}\right)\right)$, or in degree $2 n-k$, via Poincaré duality $\left(H_{d d^{\Lambda}}^{0}\left(M / \mathcal{F}_{A}\right)\right)$, or it can be indeed unprovoked by any of these $\left(H_{d+d^{\Lambda}}^{1}(M /\right.$ $\left.\mathcal{F}_{A}\right)$ ).

Remark 2 The transversally holomorphic example exhibits infinite dimensional basic de Rham, Dolbeault, Bott-Chern and Aeppli cohomologies. The basic Aeppli cohomology is infinite dimensional in bidegrees $(2,0),(1,1),(0,2)$, and $(2,2)$. In bidegree $(1,1)$, both the basic Dolbeault cohomology and its adjoint counterpart (the basic $\partial$-cohomology) are finite dimensional. This shows that also the basic Aeppli cohomology can be infinite dimensional without help from basic Dolbeault cohomology and Proposition 2. The same thing happens for the basic Bott-Chern cohomology in bidegrees $(2,1)$ and $(1,2)$. We also note that while infinite dimension of $H_{A}^{1,1}\left(M / \mathcal{F}_{A}\right)$ could be perhaps linked to the infinite dimension of $H^{2}\left(M / \mathcal{F}_{A}\right)$, it is not the case for either $H_{B C}^{2,1}\left(M / \mathcal{F}_{A}\right)$ or $H_{B C}^{1,2}\left(M / \mathcal{F}_{A}\right)$, since $H^{3}\left(M / \mathcal{F}_{A}\right)$ is finite dimensional.

Remark 3 The example proves that Poincaré duality between Bott-Chern and Aeppli cohomology may fail in the absence of the Riemannian metric. We also point out that for the same reason the Dolbeault cohomology of the example does not exhibit Serre duality. 
Remark 4 It is also worth pointing out that the basic de Rham cohomology of this example is infinite dimensional in degrees 2 and 4, but reverts to finite dimension in degree 3. To the extent of our knowledge, such an example have not been described before.

Remark 5 The property of reverting to finite dimension is important for the further developments in this field. The richest geometry in the transversally symplectic setting is the Kähler structure, and short of that - the hard Lefschetz property, that

$$
H^{n-k}\left(M / \mathcal{F}_{A}\right) \stackrel{\wedge \omega^{k}}{\longrightarrow} H^{n+k}\left(M / \mathcal{F}_{A}\right)
$$

is an epimorphism. It is a theorem that this property forces the map

$$
H_{d+d^{\Lambda}}^{\bullet}\left(M / \mathcal{F}_{A}\right) \ni[\alpha] \mapsto[\alpha] \in H^{\bullet}\left(M / \mathcal{F}_{A}\right)
$$

to be epimorphic as well, cf. $[5,14]$. It is natural to ask for examples where the infinite dimension of the former is derived from infinite dimension of the latter via the hard Lefschetz property. However, it is well known that $H^{0}\left(M / \mathcal{F}_{A}\right)$ and $H^{1}\left(M / \mathcal{F}_{A}\right)$ are always finite dimensional and there is no known example of infinite dimensional basic cohomology without the infinite dimension in the top degree.

Open Access This article is distributed under the terms of the Creative Commons Attribution 4.0 International License (http://creativecommons.org/licenses/by/4.0/), which permits unrestricted use, distribution, and reproduction in any medium, provided you give appropriate credit to the original author(s) and the source, provide a link to the Creative Commons license, and indicate if changes were made.

\section{References}

1. Angella, D.: Cohomological Aspects in Complex Non-Kähler Geometry. Springer, Berlin (2014)

2. Angella, D.: Cohomologies of certain orbifolds. J. Geom. Phys. 71, 117-126 (2013)

3. Angella, D., Dloussky, G., Tomassini, A.: On Bott-Chern cohomology of compact complex surfaces. Ann. Mat. Pura Appl. (4) 195(1), 199-217 (2016)

4. Angella, D., Tomassini, A.: On the $\partial \bar{\partial}$-Lemma and Bott-Chern cohomology. Invent. Math. 192(1), 71-81 (2013)

5. Bạk, Ł., Czarnecki, A.: A remark on the Brylinski conjecture for orbifolds. J. Aust. Math. Soc. 91(01), 1-12 (2011)

6. El Kacimi-Alaoui, A.: Opérateurs transversalement elliptiques sur un feuilletage riemannien et applications. Compos. Math. 73, 57-106 (1990)

7. Ghys, E.: Un feuilletage analytique dont la cohomologie basique est de dimension infinie. Publ. de l'IRMA de Lille, VII (1985)

8. He, Z.: Odd dimensional symplectic manifolds. MIT Ph.D. thesis. http://hdl.handle.net/1721.1/60189 (2010)

9. Ida, C., Popescu, P.: Coeffective basic cohomologies of K-contact and Sasakian manifolds. Mich. Math. J. 64(4), 797-817 (2015)

10. Mathieu, O.: Harmonic cohomology classes of symplectic manifolds. Comment. Math. Helvetici 70, 1-9 (1995)

11. Molino, P.: Riemannian Foliations. Birkhäuser, Basel (1986)

12. Raźny, P.: The Frölicher-type inequalities of foliations. J. Geom. Phys. 114, 593-606 (2017)

13. Schwarz, G.W.: On the de Rham cohomology of the leaf space of a foliation. Topology 13, 185-187 (1974)

14. Tseng, L.-S., Yau, S.-T.: Cohomology and Hodge theory on symplectic manifolds: I. J. Differ. Geom 91(3), 383-416 (2012) 